\title{
Diffusivité du joint de grain durant la précipitation discontinue de solutions solides sursaturées Mg-Al et Mg-Al-Cd
}

\author{
D. Bradai, B. Alili et M. Kadi-Hanifi \\ Faculté des Sciences (Physiques), USTHB, BP. 32, El-Alia, Dar-El-Beida, Alger, Algeria
}

\begin{abstract}
The grain boundary chemical diffusivity triple product $s \delta D_{b}$ parameter ( $s$ being the Gibbs segregation factor, $\delta$ the thickness and $\mathrm{D}_{b}$ the chemical diffusivity of the grain boundary) of $\mathrm{Al}$ in a binary $\mathrm{MgAl}$ and a ternary $\mathrm{MgAlCd}$ solid solutions during the discontinuous precipitation reaction have been determined using several analytical models. A comparison of the values obtained with the experimental data and the deduction of the activation energy confirm the reaction to be controlled by diffusion of $\mathrm{Al}$ atoms at the migrating grain boundary.
\end{abstract}

\section{INTRODUCTION}

La réaction de précipitation discontinue appartient au groupe des transformations de phases à l'état solide qui sont contrôlées par un processus de diffusion des espèces chimiques au joint de grain. Ce joint de grain en migration constitue alors le front de réaction (FR) qui sépare la région transformée de la région non encore transformée. La diffusivité chimique, exprimée comme étant le triple produit $\mathrm{s} \mathrm{D}_{\mathrm{b}}$ où $\mathrm{s}$ est le facteur de ségrégation, $\delta$ l'épaisseur du joint et $\mathrm{D}_{\mathfrak{z}}$ le coefficient de diffusion au joint, déterminée par des études de cinétique de réaction a toujours été trouvée inférieure de plusieurs ordres à celle correspondant aux joints de grains stationnaires mesurée par les méthodes de diffusion des éléments radiotraceurs. La détermination de cette diffusivité est fondamentale pour la prévision des comportements de beaucoup de matériaux métalliques usuels qui ont un intérêt commercial, spécialement dans les structures où il y a peu de données expérimentales.

Dans des travaux antérieurs [1,2], nous avions déterminé l'évolution thermique de la diffusivité chimique du joint dans le système $\mathrm{Mg}-\mathrm{Al}$ et $\mathrm{Mg}-\mathrm{Al}-\mathrm{Cd}$ lors de la réaction de précipitation discontinue en utilisant uniquement un seul modèle qui est le modèle de Petermann et Hornbogen [3] . Dans ce travail nous testons et comparons les résultats obtenus en utilisant divers autres modèles tels que celui de Turnbull [4], Aaronson et Liu [5] et Sundquist [6].

\section{EXPERIMENTAL ET PRESENTATION DES MODELES}

La majorité du protocole expérimental a été largement présentée dans les références [1,2]. Notons que les alliages étudiés ont pour compositions nominales 8,10 et $12 \mathrm{Al}$ pour les binaires et $8,10 \mathrm{Al}-0.5 \mathrm{Cd} \%$ en poids pour les ternaires.

Les différentes modélisations de la cinétique de la réaction de précipitation discontinue ont été présentées et discutées par plusieurs auteurs [7,8]. Par leur simplicité, les modèles de Turnbull (T), Aaronson et Liu (A-L) et enfin Petermann et Hornbogen $(\mathrm{P}-\mathrm{H})$ ont été les plus utilisés pour la détermination de l'évolution thermique de la diffusivité chimique lors de la précipitation discontinue. Ces modèles basés sur une simple estimation du flux de soluté sont très aisés à manipuler. En général ils aboutissent tous à une expression de la diffusivité qui s'écrit :

$$
\mathrm{s} \delta \mathrm{D}_{\mathrm{b}}=\mathrm{F} \mathrm{v} \lambda^{2}
$$


Où $v$ et $\lambda$ sont les vitesses du front de réaction et espacement interlamellaire (les deux paramètres les plus importants de la cinétique) du produit obtenu par réaction discontinue, $F$ étant un coefficient de proportionnalité qui rend compte du partage physico-chimique (troisième terme important de la cinétique) des atomes de soluté entre phases présentes. Le tableau 1 donne les différentes valeurs de $\mathrm{F}$ selon les modèles.

Tableau 1 : Différentes valeurs du coefficient F de l'équation (1).

\begin{tabular}{|c|c|c|c|}
\hline Modèle & $\mathrm{T}$ & $\mathrm{A}-\mathrm{L}$ & $\mathrm{P}-\mathrm{H}$ \\
\hline $\mathrm{F}$ & $\mathrm{X}_{\mathrm{e}} /\left(\mathrm{X}_{\mathrm{e}}-\mathrm{X}_{\mathrm{a}}\right)$ & $\left(\mathrm{X}_{\mathrm{b}}-\mathrm{X}_{\mathrm{e}}\right) / 4 *\left(\mathrm{X}_{\mathrm{b}}-\mathrm{X}_{\mathrm{a}}\right)$ & $(\mathrm{RT} / 8(-\Delta \mathrm{G}))$ \\
\hline
\end{tabular}

Ici $\mathrm{X}_{\mathrm{e}}, \mathrm{X}_{\mathrm{a}}$ et $\mathrm{X}_{\mathrm{b}}$ sont respectivement les concentrations en atomes de soluté des phases mères $\alpha_{0}$ et des phases produites $\alpha$ (phase appauvrie en atomes de soluté) et $\beta$ (généralement phase intermétallique).Les valeurs de $\mathrm{X}_{c}$ (solvus stable) et $\mathrm{X}_{\mathrm{b}}$ (composition de l'intermétallique $\mathrm{Mg}_{17} \mathrm{Al}_{12}$ ) ont été déterminées à partir du diagramme de phase du système $\mathrm{Mg}$ - $\mathrm{Al}$ [9]. $\Delta \mathrm{G}$ est la variation de l'énergie libre de Gibbs qui accompagne la réaction jusqu'à l'équilibre. Cette grandeur, qui dépend principalement des activités des différentes phases présentes, des concentrations susmentionnées ainsi que d'un terme dû à la création d'interfaces, peut être estimée par différents modèles thermodynamiques [10,11]. Le tableau 2 donne l'évolution en fonction de la température de la concentration résiduelle $X_{a}$ de la phase appauvrie en atomes de soluté et de la variation de l'énergie de Gibbs associée à la réaction de précipitation discontinue pour les trois alliages binaires. Le tableau 3 donne l'évolution en fonction de la température de la vitesse du front de réaction $v$ (en $\mu \mathrm{m} / \mathrm{h}$ ) et de l'espacement interlamellaire $\lambda$ (en $\mu \mathrm{m}$ ) durant la réaction de précipitation discontinue pour les alliages binaires $\mathrm{Mg}-\mathrm{Al}$ et ternaires $\mathrm{Mg}-\mathrm{Al}-\mathrm{Cd}$.

Tableau 2: Evolution de la concentration résiduelle $X_{a}$ de la phase appauvrie en atomes de soluté et de la variation de l'énergie de Gibbs associée à la réaction de précipitation discontinue pour les trois alliages binaires en fonction de la température.

\begin{tabular}{|c|c|c|c|c|}
\hline \multirow{2}{*}{$T(K)$} & \multirow{2}{*}{$\mathrm{X}_{\mathrm{a}}(\mathrm{x} 0.01)$} & \multicolumn{3}{|c|}{$-\Delta \mathrm{G}(\mathrm{J} / \mathrm{mole})$} \\
\hline & & $8 \% \mathrm{Al}$ & $10 \% \mathrm{Al}$ & $12 \% \mathrm{Al}$ \\
\hline 423 & 2.47 & 143.3 & 238.8 & 337.5 \\
\hline 448 & 2.85 & 120.4 & 208.2 & 306.3 \\
\hline 473 & 3.25 & 91.6 & 168.8 & 256.8 \\
\hline 498 & 3.75 & 64.1 & 128.9 & 204.3 \\
\hline 523 & 4.30 & 44.5 & 100.5 & 168.5 \\
\hline 548 & 5.02 & 22.5 & 65.1 & 117.6 \\
\hline 573 & 5.75 & 3.4 & 32.3 & 79.9 \\
\hline 598 & 655 & - & 25.2 & 50.1 \\
\hline 623 & 7.72 & - & 1.86 & 26.5 \\
\hline
\end{tabular}

Un autre modèle relativement peu utilisé pour la détermination de la diffusivité a été élaboré par Sundquist et est basé non sur une estimation du flux de soluté mais sur un bilan thermodynamique local de la réaction de précipitation discontinue. Ce modèle permet d'obtenir directement l'énergie d'activation $\mathrm{Q}_{\mathrm{B}}$ de la réaction, il s'écrit :

$$
\mathrm{v} / \Delta \mathrm{T}^{3} \sim \exp \left(\mathrm{Q}_{\mathrm{B}} / \mathrm{RT}\right)
$$

Où $\Delta \mathrm{T}$ est la différence $\mathrm{T}_{\mathrm{sv}}-\mathrm{T}$ où $\mathrm{T}_{\mathrm{sv}}$ est la température du solvus stable.

Dans le cas des systèmes ternaires, à cause des complications dues aux interactions entre les éléments d'addition avec les éléments binaires et les joints de grains, le modèle le plus utilisé est le modèle d'Aaronson et Liu en posant le facteur de ségrégation égal à 1 c'est à dire en calculant la diffusivité réduite $\delta D_{b}$ et en faisant l'approximation $\mathrm{X}_{\mathrm{a}}=\mathrm{X}_{\mathrm{e}}$. 
Tableau 3 : Evolution de la vitesse du front de réaction $v($ en $\mu \mathrm{m} / \mathrm{h}$ ) et de l'espacement interlamellaire $\lambda$ (en $\mu \mathrm{m}$ ) en fonction de la température durant la réaction de précipitation discontinue pour les alliages binaires $\mathrm{Mg}$ - $\mathrm{Al}$ et ternaires $\mathrm{Mg}-\mathrm{Al}-\mathrm{Cd}$.

\begin{tabular}{|c|c|c|c|c|c|c|c|c|c|c|}
\hline \multirow{2}{*}{$\mathrm{T}(\mathrm{K})$} & \multicolumn{2}{|c|}{$8 \mathrm{Al}$} & \multicolumn{2}{c|}{$10 \mathrm{Al}$} & \multicolumn{2}{c|}{$12 \mathrm{Al}$} & \multicolumn{2}{c|}{$8 \mathrm{Al}-5 \mathrm{Cd}$} & \multicolumn{2}{c|}{$10 \mathrm{Al}-5 \mathrm{Cd}$} \\
\cline { 2 - 11 } & $\mathrm{v}$ & $\lambda$ & $\mathrm{v}$ & $\lambda$ & $\mathrm{v}$ & $\lambda$ & $\mathrm{V}$ & $\lambda$ & $\mathrm{v}$ & $\lambda$ \\
\hline 423 & 0.48 & 0.55 & 1.47 & 0.34 & 2.13 & 0.21 & 0.51 & 0.42 & 0.84 & 0.35 \\
\hline 448 & 1.74 & 0.57 & 7.3 & 0.37 & 9.90 & 0.26 & 1.46 & 0.50 & 7.84 & 0.39 \\
\hline 473 & 7.90 & 0.56 & 19.7 & 0.47 & 31.0 & 0.27 & 7.90 & 0.61 & 15.3 & 0.40 \\
\hline 498 & 23.7 & 0.65 & 49.4 & 0.55 & 65.1 & 0.25 & 7.87 & 0.81 & 42.3 & 0.52 \\
\hline 523 & 33.9 & 0.69 & 78.3 & 0.59 & 128 & 0.31 & 14.9 & 0.91 & 50.8 & 0.58 \\
\hline 548 & 56.5 & 0.72 & 101 & 0.62 & 175 & 0.42 & 38.5 & 1.21 & 62.5 & 0.67 \\
\hline 573 & 99.0 & 0.83 & 110 & 0.70 & 234 & 0.51 & 40.3 & 1.42 & 108 & 0.81 \\
\hline 598 & - & - & 125 & 0.74 & 288 & 0.55 & - & - & 114 & 0.91 \\
\hline 623 & - & - & 142 & 0.79 & 345 & 0.58 & - & - & 164 & 1.20 \\
\hline
\end{tabular}

\section{RESULTATS ET INTERPRETATIONS}

La figure 1 montre l'évolution de la diffusivité chimique en fonction de l'inverse de la température absolue de revenu pour le système binaire $\mathrm{Mg}-\mathrm{Al}$ et de la diffusivité réduite pour le système ternaire $\mathrm{Mg}$ $\mathrm{Al}-\mathrm{Cd}$. Pour le premier système, les valeurs obtenues en utilisant les modèles de Turnbull et Petermann et Hornbogen sont très rapprochées, celles obtenues en utilisant le modèle d'Aaronson et Liu sont inférieures alors que celles obtenues en utilisant le modèle de Sundquist sont nettement supérieures. Ces différences sont dues essentiellement aux approches phénoménologiques sur lesquelles sont basées les modèles. Pour le deuxième système, les valeurs restent très rapprochées pour les deux concentrations étudiées et dans le même ordre de grandeur que celles du système binaire.

Nous avons déterminé les énergies d'activation $Q_{B}$ de la diffusion chimique au joint des atomes de soluté à partir des pentes des courbes d'évolution de la diffusivité chimique en fonction de l'inverse de la température absolue de revenu (tableau 4). On peut noter que les valeurs de l'énergie d'activation obtenues par différents modèles sont presque les mêmes (dans une fourchette de $10 \%$ ) pour le même alliage hormis pour le modèle de Sundquist.

Les modèles de Petermann et Hornbogen et Sundquist aboutissent aux plus grandes valeurs. On peut aussi noter de manière surprenante que les valeurs de l'énergie d'activation pour les alliages ternaires sont relativement inférieures à celles pour les binaires. Ce qui suppose donc un accroissement de la précipitation discontinue, ce qui n'est pas du tout le cas d'après nos observations où la tendance était plus vers le ralentissement, voire la disparition totale de la réaction de précipitation discontinue aux hautes températures.

Tableau 4: Energies d'activation $\mathrm{Q}_{\mathrm{b}}$ de la diffusion chimique au joint des atomes de soluté lors la réaction de précipitation discontinue pour les alliages binaires $\mathrm{Mg}-\mathrm{Al}$ et ternaires $\mathrm{Mg}$ - $\mathrm{Al}-\mathrm{Cd}$.

\begin{tabular}{|l|c|c|c|c|}
\hline \multirow{2}{*}{ Alliage } & \multicolumn{4}{|c|}{$\mathrm{QB}_{\mathrm{B}}(\mathrm{kJ} / \mathrm{mol})$} \\
\cline { 2 - 5 } & $\mathrm{P}-\mathrm{H}$ & $\mathrm{T}$ & $\mathrm{A}-\mathrm{L}$ & $\mathrm{S}$ \\
\hline $\mathrm{Mg} 8 \mathrm{Al}$ & 115.9 & 99.3 & 93.3 & 123.4 \\
\hline $\mathrm{Mg} 10 \mathrm{Al}$ & 115.6 & 94.3 & 99.5 & 107.2 \\
\hline $\mathrm{Mg} 12 \mathrm{Al}$ & 103.7 & 97.1 & 109.9 & 96.8 \\
\hline $\mathrm{Mg} 8 \mathrm{Al} 0.5 \mathrm{Cd}$ & - & - & 92.0 & - \\
\hline $\mathrm{Mg} 10 \mathrm{Al} 0.5 \mathrm{Cd}$ & - & - & 70.6 & - \\
\hline
\end{tabular}

Les seules données disponibles concernant la diffusion en volume de $\mathrm{Al}$ dans $\mathrm{Mg}$ sont celles publiées par Moreau et al [12]. Ces auteurs ont déterminé le coefficient de diffusion de $\mathrm{Al}$ dans $\mathrm{Mg}$ et l'énergie d'activation correspondante : le facteur pré-exponentiel est égal à $1210^{4}\left(\mathrm{~m}^{2} / \mathrm{s}\right)$ et $\mathrm{Q}=144$ $\mathrm{kJ} / \mathrm{mol})$. En utilisant ces paramètres, le coefficient de diffusion en volume vaut entre $10^{-19}$ et $10^{-15}\left(\mathrm{~m}^{2} / \mathrm{s}\right)$ pour des températures comprises entre 475 et $625 \mathrm{~K}$; ces valeurs sont plus faibles (approximativement de 7 ordres de grandeur) que celles du coefficient de diffusion $\mathrm{D}_{\mathrm{b}}$ au joint de grain en migration durant la 

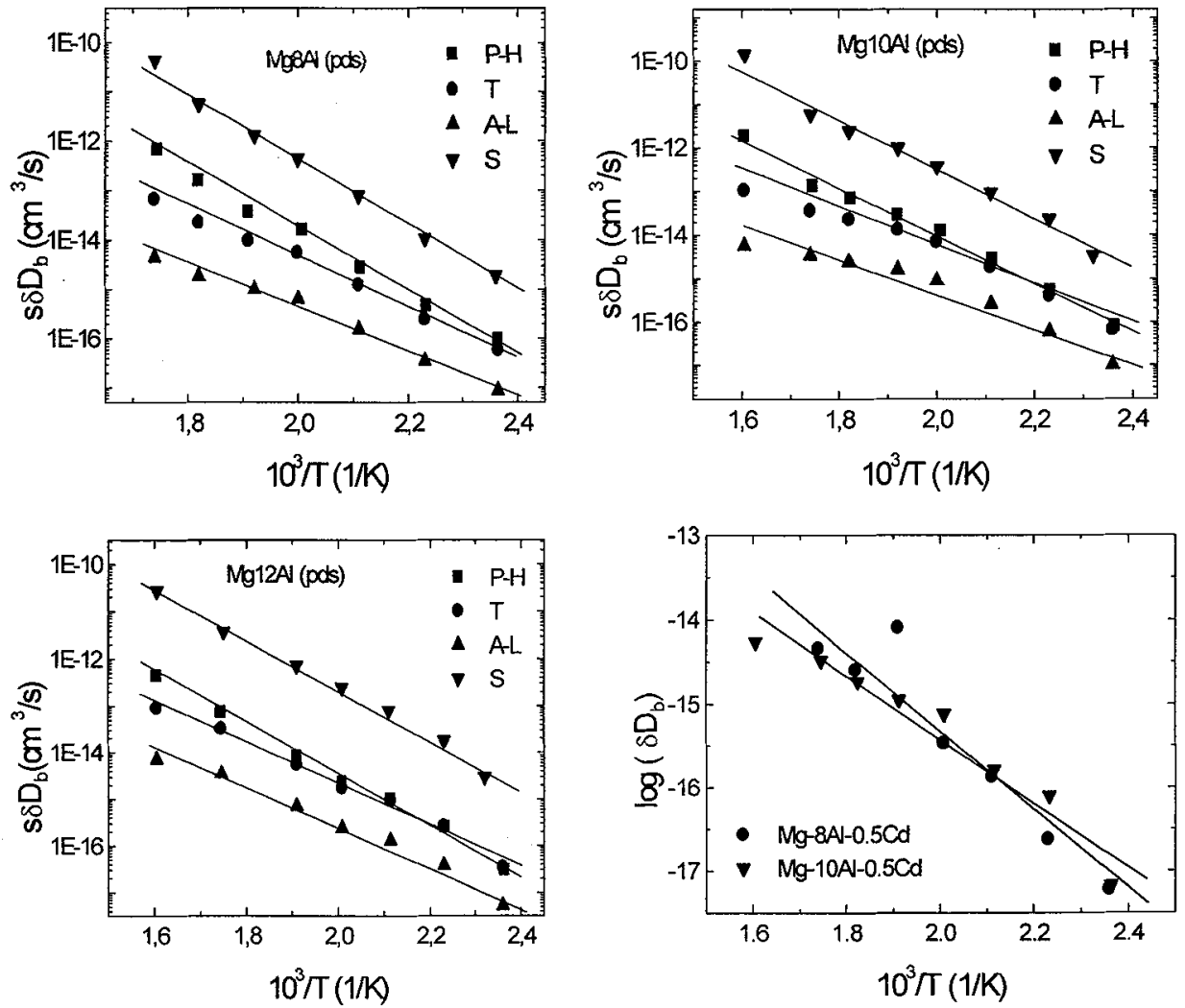

Figure 1 : Evolution de la diffusivité chimique du joint de grain pour l'alliage binaire $\mathrm{Mg}-\mathrm{Al}$ et de la diffusivité chimique réduite pour l'alliage ternaire $\mathrm{Mg}$-Al-Cd calculée en utilisant les modèles de Petermann-Hornbogen (P-H), Turnbull (T), Aaronson-Liu (A-L) et enfin Sundquist (S).

réaction de précipitation discontinue aux mêmes températures (en prenant $s=1$ et $\delta=0.5 \mathrm{~nm}$ ). Par conséquent, on peut conclure que la précipitation discontinue dans les systèmes $\mathrm{Mg}-\mathrm{Al}$ et $\mathrm{Mg}-\mathrm{Al}-\mathrm{Cd}$ est gouvernée par la diffusion de Al le long du joint de grain en migration.

\section{Conclusion}

Le calcul de la diffusivité du joint de grain durant la précipitation discontinue de solutions solides $\mathrm{Mg}-\mathrm{Al}$ et $\mathrm{Mg}-\mathrm{Al}-\mathrm{Cd}$ en utilisant différents modèles phénoménologiques, montre que cette réaction est contrôlée par un processus de diffusion au joint de grain. 


\section{Références}

[1] Bradai D., Thèse de Doctorat es Sciences, Alger, Algérie (1999) pp.71-103.

[2] Bradai D., Kadi-Hanifi M, Zieba P., Kuschke W.M. et Gust W., J. Mater. Sci, 84 (1999) 5331-5336.

[3] Petermann J. et Hornbogen E., Z. Metallk., 59 (1968) 814-822.

[4] Turnbull D., Acta Metall., 3 (1955) 55-63.

[5] Aaronson H. I. et Liu Y. C., Scripta Metall., 2 (1968) 1-8.

[6] Sundquist B. E., Metall. Trans., 4A (1973) 1919-1934.

[7] Duly D., Thèse de Doctorat d'université, Grenoble, France (1992) pp.87-128.

[8] Manna I., Jha J.N. et Gust W.," Advances in Science, Technology and Applications of Zn-Al alloys" ( éditions G. Torres Villasenor, Y.H. Zhu and C. Pina., Mexico (1994) 121-126.

[9] Massalski T. B., " Binary Alloy Phase Diagrams ", (Les éditions ASM International, Material Park. Ohio, (1990) p. 170.

[10] Bögel A. et Gust W., Z. Metallk., 88 (1997) 1-8.

[11] Bradai D., Zieba P., Gust W., à soumettre à Calphad (2001).

[12] Moreau G., Cornet J.A. et Calais D., J. Nucl. Mater., 38 (1971) 197-202. 